OHSTPY-HEP-T-98-009

April 1998

\title{
Dynamical SUSY Breaking with a Hybrid Messenger Sector
}

\author{
Stuart Raby ${ }^{\dagger}$ and Kazuhiro Tobe* \\ Department of Physics, \\ The Ohio State University, \\ 174 W. 18th Ave., \\ Columbus, Ohio 43210 \\ †raby@mps.ohio-state.edu \\ *tobe@pacific.mps.ohio-state.edu
}

\begin{abstract}
In this paper we present a dynamical model of SUSY breaking with a hybrid messenger sector. SUSY is broken dynamically at a scale of order $10^{9} \mathrm{GeV}$ via strong SU(2) gauge interactions. SUSY breaking is then transmitted to the observable sector via two distinct sources: (1) messengers, carrying Standard Model gauge quantum numbers, with the messenger mass of order $10^{15} \mathrm{GeV}$, and (2) the $\mathrm{D}$ term of an anomalous $\mathrm{U}(1)_{X}$. The model is quite constrained. The messenger scale is fixed by the Fayet-Iliopoulos term for the anomalous $\mathrm{U}(1)_{X}$ interaction. In addition, we show that the D term SUSY breaking contributions to squark and slepton masses are "naturally" the same order as those coming from the messengers.
\end{abstract}




\section{Introduction}

Supersymmetry [SUSY] is a strongly motivated candidate for new physics beyond the Standard Model [SM]. The minimal supersymmetric particle content is well defined and the interactions of all the new superparticles [sparticles] are constrained by the observed SM interactions as long as the theory has a conserved $\mathrm{R}$ parity. One might naively expect that it would be a simple task to search for the new sparticles. Of course, it is not easy and the reason is two-fold. First, the masses of the new superparticles depend on how supersymmetry is broken and secondly, since these particles must be produced in high energy/luminosity collisions, the signal to background ratio is generically quite small. Thus in order to find SUSY one must look for particular signatures which may be pulled out of the SM background by skilled experimental hands. In this paper we present another scenario for obtaining squark, slepton and gaugino masses. The mechanism is a hybrid of gaugemediated SUSY breaking via messengers carrying SM gauge interactions and D-term SUSY breaking associated with an anomalous $U(1)_{X}$ gauge symme-

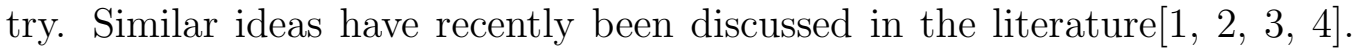
The new feature in this paper is that the SUSY breaking contributions of the messengers and that of the D term are "naturally" expected to be of the same order of magnitude. In addition the messenger scale is determined by the Fayet-Illiopoulos D term contribution to $\mathrm{U}(1)_{X}$. We discuss the spectrum of sparticle masses for this theory. Note, in recent years, anomalous $U(1)_{X}$ symmetries have also been used to construct models of fermion and sfermion masses [5 - [1].

\section{Dynamical SUSY Breaking Sector}

Consider an $\mathrm{SU}(\mathrm{N})$ gauge group with $\mathrm{N}_{F}=\mathrm{N}$ flavors. Such a theory has a quantum moduli space first considered by Seiberg[12]. Models of dynamical SUSY breaking using these theories as a starting point have also been discussed in the literature 13, 14. We shall focus on the simplest model of this type with $\mathrm{N}=\mathrm{N}_{F}=2$.

The model includes the chiral superfields $-Q_{i \alpha}, \bar{Q}^{i \alpha}$ - transforming as a $2+\overline{2}$ of the strong $\mathrm{SU}(2)$ gauge symmetry where $\mathrm{i}=1,2$ is a gauge index and $\alpha=1,2$ is a flavor index. The theory has an $\mathrm{SU}(4)$ flavor symmetry, but we 
shall break this full flavor symmetry by weakly gauging an $\mathrm{SU}(2)_{F}$ subgroup such that $Q, \bar{Q}$ transform as a $2, \overline{2}$ of $\mathrm{SU}(2)_{F}$, respectively. In addition, the theory has a global baryon number symmetry $\mathrm{U}(1)_{B}$ where $(Q, \bar{Q})$ have charge $(1,-1)$. It will be convenient to work in terms of $\mathrm{SU}(2)$ strong gauge singlet superfields defined by

$$
\begin{aligned}
M_{\alpha}^{\beta} & =\bar{Q}^{i \beta} Q_{i \alpha} \\
B_{\alpha \beta} & =\frac{1}{\sqrt{2}} Q_{i \alpha} Q_{j \beta} \epsilon^{i j}=-B_{\beta \alpha} \\
\bar{B}^{\alpha \beta} & =\frac{1}{\sqrt{2}} \bar{Q}^{i \alpha} \bar{Q}^{j \beta} \epsilon_{i j}=-\bar{B}^{\beta \alpha}
\end{aligned}
$$

The quantum moduli space is given by the equation

$$
\operatorname{det} M-\bar{B}^{\alpha \beta} B_{\alpha \beta}=\Lambda_{s}^{4}
$$

where $\Lambda_{s}$ is the dynamically determined scale of the strong $\mathrm{SU}(2)$ gauge symmetry.

The dynamical SUSY breaking sector of the theory includes, in addition to the $\mathrm{SU}(2) \times \mathrm{SU}(2)_{F}$ gauge theory with chiral states described above, the states $X, \quad A^{\alpha \beta}=-A^{\beta \alpha}, \quad \bar{A}_{\alpha \beta}=-\bar{A}_{\beta \alpha}$, and $S^{\alpha}{ }_{\beta}$ where $X, A, \bar{A}$ are $\mathrm{SU}(2) \times \mathrm{SU}(2)_{F}$ singlets and $S^{\alpha}{ }_{\beta}$ is an $\mathrm{SU}(2)$ singlet and $\mathrm{SU}(2)_{F}$ triplet. They have charge $(0,-2,2,0)$ under $\mathrm{U}(1)_{B}$, respectively. Note, we also can define the fields $S_{a}, a=1,2,3$ by $S^{\alpha}{ }_{\beta} \equiv \sqrt{2} S_{a}\left(T_{a}\right)^{\alpha}{ }_{\beta}$.

The most general superspace potential, invariant under $\mathrm{SU}(2) \times \mathrm{SU}(2)_{F} \times \mathrm{U}(1)_{B}$, for the dynamical SUSY breaking sector is given by

$$
\begin{aligned}
W= & \frac{\lambda}{\sqrt{2}} X \operatorname{Tr} M+\lambda^{\prime} S^{\alpha}{ }_{\beta} M^{\beta}{ }_{\alpha} \\
& +\lambda^{\prime \prime} A^{\alpha \beta} B_{\alpha \beta}+\bar{\lambda}^{\prime \prime} \bar{A}_{\alpha \beta} \bar{B}^{\alpha \beta} \\
& +U\left[\operatorname{det} M-\bar{B}^{\alpha \beta} B_{\alpha \beta}-\Lambda_{s}^{4}\right]
\end{aligned}
$$

where $U$ is a Lagrange multiplier enforcing quantum moduli space.

This theory breaks supersymmetry dynamically. We can study the theory for large values of the fields $X, A^{\alpha \beta}, \bar{A}_{\alpha \beta}, S^{\alpha}{ }_{\beta}$ by integrating out the states $M^{\beta}{ }_{\alpha}, \quad B_{\alpha \beta}, \quad \bar{B}^{\alpha \beta}$ and the auxiliary field $U$. We find the effective superspace potential

\footnotetext{
${ }^{1}$ Actually, since SUSY is broken the auxiliary field for $U$ gets a nonvanishing vev. If one minimizes the full potential we obtain higher order corrections in $\lambda^{2}, \lambda^{\prime 2}$ etc. to the leading order expression for $W$ below.
} 


$$
W=-2 \Lambda_{s}^{2} \sqrt{\lambda^{2} X^{2} / 2-\lambda^{\prime 2} \operatorname{Tr} S^{2} / 2-\lambda^{\prime \prime} \bar{\lambda}^{\prime \prime} A^{\alpha \beta} \bar{A}_{\alpha \beta}}
$$

and the resulting scalar potential $V=\left|\frac{\partial W}{\partial X}\right|^{2}+\operatorname{Tr}\left|\frac{\partial W}{\partial S^{\alpha}{ }_{\beta}}\right|^{2}+\left|\frac{\partial W}{\partial A^{\alpha \beta}}\right|^{2}+\left|\frac{\partial W}{\partial A_{\alpha \beta}}\right|^{2}$ is given by (see fig. 1)

$$
V=\Lambda_{s}^{4} \frac{\left(\lambda^{4}|X|^{2}+\lambda^{4}\left|S^{\alpha}{ }_{\beta}\right|^{2}+\lambda^{\prime \prime 2} \bar{\lambda}^{\prime \prime 2}\left(\left|A^{\alpha \beta}\right|^{2}+\left|\bar{A}_{\alpha \beta}\right|^{2}\right)\right.}{\left|\lambda^{2} X^{2} / 2-\lambda^{\prime 2} \operatorname{Tr} S^{2} / 2-\lambda^{\prime \prime} \lambda^{\prime \prime} A^{\alpha \beta} A_{\alpha \beta}\right|}
$$

The potential has no supersymmetric minima. There are several flat directions. For example, if we take $\left\{X, A^{\alpha \beta}, \bar{A}_{\alpha \beta}, S^{\alpha}{ }_{\beta}\right\}=\{X, 0,0,0\}$ we have

$$
V(X, 0,0,0)=2 \lambda^{2} \Lambda_{s}^{4}
$$

or for $\left\{0,0,0, S_{3}\right\}$, we have

$$
V\left(0,0,0, S_{3}\right)=2 \lambda^{\prime 2} \Lambda_{s}^{4}
$$

For the range of parameters satisfying $\lambda^{\prime}<\lambda \sim \lambda^{\prime \prime} \sim \bar{\lambda}^{\prime \prime}$ the flat direction with $\left\{X, A^{\alpha \beta}, \bar{A}_{\alpha \beta}, \quad S^{\alpha}{ }_{\beta}\right\}=\left\{0,0,0, S_{3}\right\}$ is the lowest energy state. Along this flat direction the superspace potential is given by

$$
W=-i \sqrt{2} \lambda^{\prime} S_{3} \Lambda_{s}^{2}
$$

Radiative corrections lift this tree level degeneracy. As discussed in the literature [14, the Lagrangian receives corrections when integrating out states between $S_{3}$ and $M$ ( $M$ is either the Planck scale or the string scale) a la Wilson. We find

$$
\mathcal{L}=\int d^{4} \theta \tilde{Z}_{S}\left|S_{3}\right|^{2}+\int d^{2} \theta W\left(S_{3}\right)+\text { h.c. }
$$

where at one loop

$$
\begin{aligned}
\tilde{Z}_{S}= & 1+\gamma_{S} \ln \left(M^{2} /\left|S_{3}\right|^{2}\right) \text { and } \\
\gamma_{S}= & \left(2 \lambda^{\prime 2}-4 g_{F}^{2}\right) / 16 \pi^{2}
\end{aligned}
$$

is the anomalous dimension of $S_{3}$ and $g_{F}$ is the $\mathrm{SU}(2)_{F}$ gauge coupling constant. More generally we have

$$
\gamma_{S}=-\frac{1}{2} \frac{\partial \ln \tilde{Z}_{S}}{\partial \ln \mu}
$$




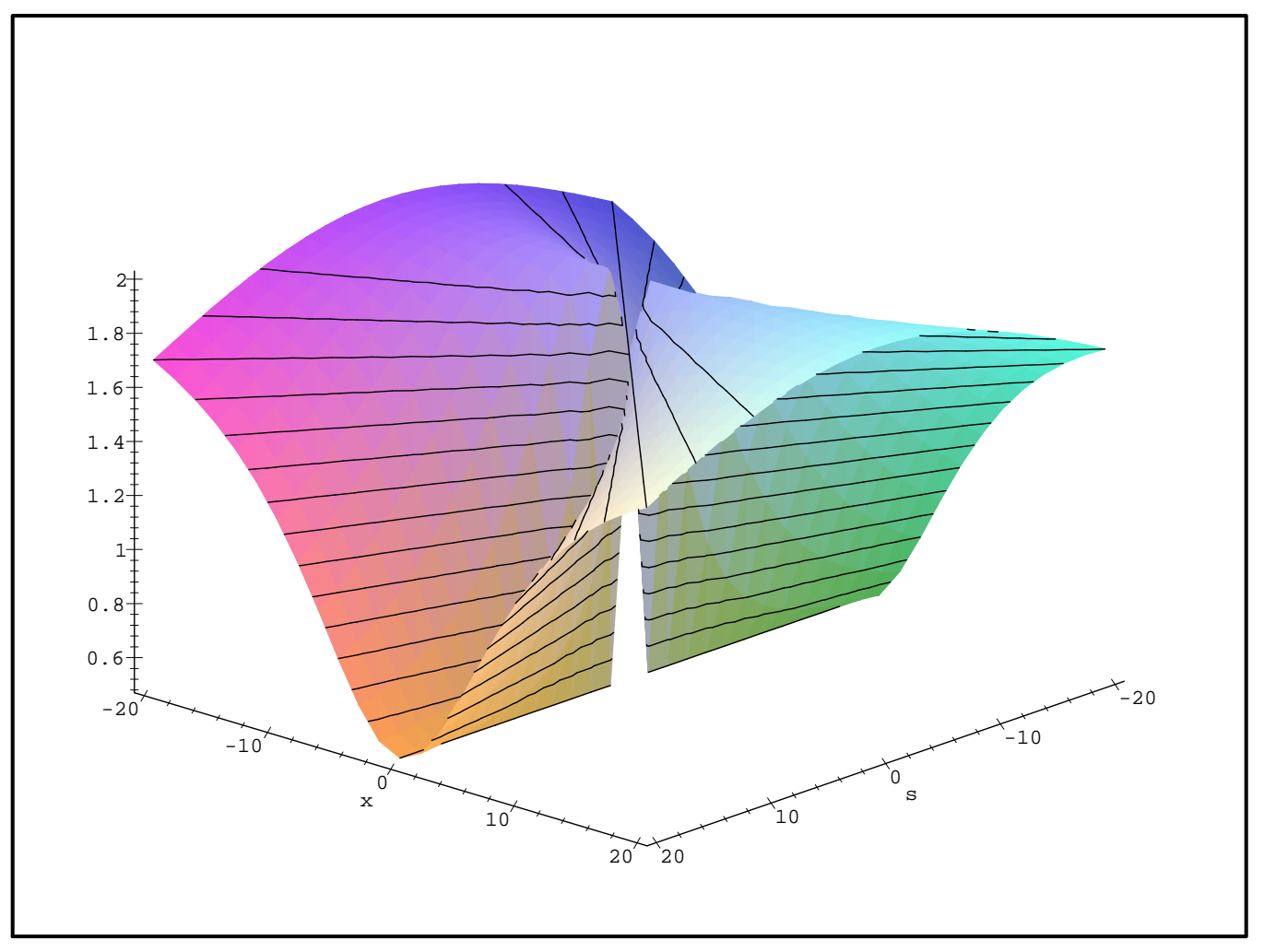

Figure 1: The scalar potential (eqn. 5). Here we assume that $A=\bar{A}=0$, $\lambda=1$, and $\lambda^{\prime}=i / 2$. 
where $\mu=\left|S_{3}\right|$ and both $\lambda^{\prime}$ and $g_{F}$ are $\mu$ dependent. Thus the scalar potential is given by

$$
V_{0}=K_{S S^{\dagger}}^{-1}\left|\frac{\partial W}{\partial S_{3}}\right|^{2}
$$

with $K\left(S, S^{\dagger}\right)=\tilde{Z}_{S}\left|S_{3}\right|^{2}$ and $K_{S S^{\dagger}} \equiv \partial^{2} K\left(S, S^{\dagger}\right) / \partial S \partial S^{\dagger}$. Hence

$$
V_{0} \approx 2 \lambda^{\prime 2} \Lambda_{s}^{4} / \tilde{Z}_{S}
$$

The extremum of the potential $\bar{S}_{0}$ is given by

$$
\begin{aligned}
S_{3} \frac{\partial V_{0}}{\partial S_{3}}= & \frac{-2 \lambda^{\prime 2} \Lambda_{s}^{4}}{\tilde{Z}_{S}} \frac{\partial \ln \tilde{Z}_{S}}{\partial \ln \left|S_{3}\right|} \\
& =2 V_{0} \gamma_{S}\left(\bar{S}_{0}\right) \quad=0
\end{aligned}
$$

This is satisfied for $\gamma_{S}\left(\bar{S}_{0}\right) \equiv 0$ or $\lambda^{\prime 2}=2 g_{F}^{2}$.

We can check that this is in fact a minimum. Consider the second derivative

$$
\left|S_{3}\right|^{2} \frac{\partial^{2} V_{0}}{\partial\left|S_{3}\right|^{2}}=2 V_{0} \frac{\partial \gamma_{S}\left(S_{3}\right)}{\partial \ln \left|S_{3}\right|} \quad \text { at } \mathrm{S}_{3}=\overline{\mathrm{S}}_{0}
$$

We may obtain $\gamma_{S}\left(S_{3}\right)$ in an expansion in $\ln \left(\left|S_{3}\right| /\left|\bar{S}_{0}\right|\right)$. Using the definitions of the beta functions

$$
\begin{array}{rc}
\beta_{\lambda^{\prime}} \equiv \frac{\partial \lambda^{\prime}}{\partial \ln \mu} & =\lambda^{\prime}\left(\gamma_{S}+\gamma_{Q}+\gamma_{\bar{Q}}\right) \\
\beta_{g_{F}} \equiv \frac{\partial g_{F}}{\partial \ln \mu} & =-b g_{F}^{3} / 16 \pi^{2} \\
\text { with } & b=3 C_{2}(S U(2))-T(S)-2 T(Q)-2 T(\bar{Q})=2,
\end{array}
$$

and eqn. 11 for $\gamma_{S}$, we find to first order in $\ln \left(\left|S_{3}\right| /\left|\bar{S}_{0}\right|\right)$

$$
\begin{gathered}
\gamma_{S}\left(S_{3}\right) \approx \gamma_{S}\left(\bar{S}_{0}\right)+2\left(\frac{\lambda^{\prime} \beta_{\lambda^{\prime}(}\left(\bar{S}_{0}\right)}{8 \pi^{2}} \ln \left(\left|S_{3}\right| /\left|\bar{S}_{0}\right|\right)-\frac{2 g_{F} \beta_{g_{F}}\left(\bar{S}_{0}\right)}{8 \pi^{2}} \ln \left(\left|S_{3}\right| /\left|\bar{S}_{0}\right|\right)\right) \text { or } \\
\gamma_{S}\left(S_{3}\right) \quad \approx 2\left(\frac{\lambda^{\prime 2}\left(\bar{S}_{0}\right)}{8 \pi^{2}}\left(\gamma_{Q}\left(\bar{S}_{0}\right)+\gamma_{\bar{Q}}\left(\bar{S}_{0}\right)\right)+4 b\left(\frac{\alpha_{F}}{4 \pi}\right)^{2}\right) \ln \left(\frac{\left|S_{3}\right|}{\left|S_{0}\right|}\right)
\end{gathered}
$$

Thus

$$
\begin{gathered}
\frac{\partial \gamma_{S}\left(S_{3}\right)}{\partial \ln \left|S_{3}\right|}=2\left(\frac{\lambda^{\prime 2}\left(\bar{S}_{0}\right)}{8 \pi^{2}}\left(\gamma_{Q}\left(\bar{S}_{0}\right)+\gamma_{\bar{Q}}\left(\bar{S}_{0}\right)\right)+4 b\left(\frac{\alpha_{F}}{4 \pi}\right)^{2}\right) \\
\text { at } S_{3}=\bar{S}_{0}
\end{gathered}
$$


Now

$$
\gamma_{Q}=\gamma_{\bar{Q}}=\frac{3}{2}\left(\lambda^{\prime 2}-g_{F}^{2}-g^{2}\right) / 16 \pi^{2}
$$

and at $\bar{S}_{0}$ we have $\lambda^{\prime 2}=2 g_{F}^{2}$, hence

$$
\gamma_{Q}\left(\bar{S}_{0}\right)=\gamma_{\bar{Q}}\left(\bar{S}_{0}\right)=\frac{3}{2}\left(g_{F}\left(\bar{S}_{0}\right)^{2}-g\left(\bar{S}_{0}\right)^{2}\right) / 16 \pi^{2} .
$$

Thus it is sufficient for $g<g_{F}$ at $\bar{S}_{0}$ and $b \geq 0$ for the second derivative of $V_{0}$ to be positive. Although the $\mathrm{SU}(2)$ coupling $g$ is necessarily large near $\Lambda_{s}$, and larger than the flavor coupling $g_{F}$, it varies more quickly than $g_{F}$ and can easily be less than $g_{F}$ at $\bar{S}_{0}$. We have $\alpha\left(\bar{S}_{0}\right)=g\left(\bar{S}_{0}\right)^{2} / 4 \pi=\pi /\left(2 \ln \left(\bar{S}_{0} / \Lambda_{s}\right)\right)$. Thus for $\Lambda_{s} \sim 10^{9} \mathrm{GeV}, \bar{S}_{0} \sim 10^{14} \mathrm{GeV}$ we find $\alpha\left(\bar{S}_{0}\right) \sim .14$. If $g<g_{F}$ at $\bar{S}_{0}, g_{F}$ can still be perturbative and since it varies more slowly than $g$ it only gets strong at a scale $\Lambda_{F}<<\Lambda_{s}$. With these caveats, the scalar potential $V_{0}$ has a minimum at $S_{3}=\bar{S}_{0}$ defined by $\gamma_{S}\left(\bar{S}_{0}\right)=0$.

We assume that $\bar{S}_{0}$ is at an intermediate scale of order $10^{14-15} \mathrm{GeV}$. The precise value is not important. We also assume that $S^{\alpha}{ }_{\beta}$ couples to messengers which carry SM gauge quantum numbers. In the minimal messenger model we would have a coupling of the form

$$
W \supset \frac{1}{M} \operatorname{Tr}\left(S_{\beta}^{\alpha}\right)^{2} \overline{5} 5
$$

where $5, \overline{5}$ are messengers transforming as indicated in $S U(5)$ representations: Note, however, this is not necessarily a grand unified model, since we only need to gauge the $S U(3) \times S U(2) \times U(1)$ subgroup of $S U(5)$.

Messengers contribute masses to squarks and sleptons proportional to their SM quantum numbers. At two loops we have

$$
\tilde{m}^{2}=8 \frac{\left|F_{S_{3}}\right|^{2}}{<S_{3}>^{2}}\left[\sum_{i=1}^{3} C_{i}\left(\frac{\alpha_{i}\left(M_{\text {mess }}\right)}{4 \pi}\right)^{2}\right]
$$

where $<S_{3}>=\bar{S}_{0}, C_{3}=\frac{4}{3}$ for color triplets and zero for singlets, $C_{2}=\frac{3}{4}$ for weak doublets and zero for singlets, and $C_{1}=\frac{3}{5}\left(\frac{Y}{2}\right)^{2}$, with the ordinary hypercharge $Y$ normalized as $Q=T_{3}+\frac{1}{2} Y$ and $\alpha_{1}$, GUT normalized.

\footnotetext{
${ }^{2}$ Note, the messenger mass $M_{\text {mess }}=<S_{3}>^{2} / M$ and we may define an effective SUSY breaking scale $F=2 F_{S_{3}}<S_{3}>/ M$. The scale $M$ in the higher dimension operator drops out in the ratio $F / M_{\text {mess }}=2 F_{S_{3}} /<S_{3}>\equiv \Lambda \sim 10^{5} \mathrm{GeV}$, where $\Lambda$ is the effective SUSY breaking scale in the observable sector.
} 
Gauginos obtain mass at one loop given by

$$
M_{i}=\frac{\alpha_{i}\left(M_{m e s s}\right)}{4 \pi} \Lambda \quad(\text { for } i=1,2,3) .
$$

\section{Adding an Anomalous $\mathrm{U}(1)_{X}$ to the theory}

Now consider how the theory changes if we include an anomalous $\mathrm{U}(1)_{X}$ gauge interaction. Such interactions are natural in strings. The scalar potential given by

$$
V=V_{0}+\frac{1}{2} D_{X}^{2}
$$

now includes the contribution of the auxiliary field $D_{X}$ where

$$
D_{X}=g_{X}\left(\sum_{a} Q_{a}^{X}\left|\phi_{a}\right|^{2}+\xi\right)
$$

and

$$
\xi=\epsilon M_{P l}^{2}
$$

where

$$
\epsilon=\frac{g_{X}^{2} \operatorname{Tr} \mathbf{Q}^{\mathbf{X}}}{192 \pi^{2}}\left(\frac{\sqrt{2} M_{s t}}{g_{X} M_{P l}}\right)^{2} .
$$

$\xi$ is the Fayet-Iliopoulos D term which is generated at one loop [15, 16]. $M_{s t}$ is the string scale associated with the scale of compactification and $M_{P l}=$ $2.4 \times 10^{18} \mathrm{GeV}$ is the reduced Planck scale. Note in weak coupling string theory $M_{s t}=g_{X} M_{P l} / \sqrt{2}$.

We assume that $\operatorname{Tr} \mathbf{Q}^{\mathbf{X}}>0$ and that $Q_{S}^{X}<0$ so that the $\mathrm{D}$ term minimization condition $D_{X}\left(\phi_{a}\right)=0$ has the solution $\phi_{a}=0$ for all $a$ EXCEPT for $S_{3} \equiv S_{0}$ with $S_{0}=\sqrt{\xi /\left|Q_{S}^{X}\right|}$. Note, in general, $S_{0} \neq \bar{S}_{0}$ discussed earlier. Thus the true minimum of $V$ is neither at $S_{0}$ nor at $\bar{S}_{0}$ and at the minimum there will be a non-zero vacuum value for $D_{X}$. We now show that the $\mathrm{D}$ term contribution to sparticle masses is comparable to the SUSY breaking contribution from the messenger sector as discussed above. Moreover the D term also sets the messenger scale. This is the main result of the paper.?

\footnotetext{
${ }^{3}$ Note, once the anomalous $\mathrm{U}(1)_{X}$ is included the fields $\left\{X, A^{\alpha \beta}, \bar{A}_{\alpha \beta}\right\}$ are no
} 
The D term introduces the largest amount of curvature in the potential for $S_{3}$. Hence let $\left\langle S_{3}\right\rangle=S_{0}+\delta S$ where $\delta S$ is a small correction. By minimizing $V$ and treating $\delta S$ perturbatively, we find

$$
\delta S=-\frac{1}{4\left(Q_{S}^{X}\right)^{2} g_{X}^{2} S_{0}^{3}}\left(\left.S_{0} \frac{\partial V_{0}}{\partial S_{3}}\right|_{S_{3}=S_{0}}\right)
$$

and a shift in $D_{X}$ given by

$$
g_{X}<D_{X}>=-2 g_{X}^{2}\left|Q_{S}^{X}\right| S_{0} \delta S
$$

The SUSY breaking correction to scalar masses from the D term is given by

$$
\delta \tilde{m}_{a}^{2}=g_{X}<D_{X}>Q_{a}^{X}
$$

Using the result for $\left(\left.S_{0} \frac{\partial V_{0}}{\partial S_{3}}\right|_{S_{3}=S_{0}}\right)$ (eqn. 15) and $V_{0}\left(S_{0}\right)=\left|F_{S_{3}}\right|^{2}$, we have

$$
\begin{aligned}
\delta \tilde{m}_{a}^{2} & =Q_{a}^{X} \frac{1}{\left|Q_{S}^{X}\right| S_{0}^{2}} V_{0}\left(S_{0}\right) \gamma_{S}\left(S_{0}\right) \\
& =\left(Q_{a}^{X} /\left|Q_{S}^{X}\right|\right) \frac{\left|F_{S_{3}}\right|^{2}}{S_{0}^{2}} \gamma_{S}\left(S_{0}\right)
\end{aligned}
$$

Note, as discussed previously, $\gamma_{S}\left(\bar{S}_{0}\right) \equiv 0$ and in eqn. 18 we have obtained $\gamma_{S}\left(S_{3}\right)$ in an expansion in $\ln \left(\left|S_{3}\right| /\left|\bar{S}_{0}\right|\right)$. Hence $\gamma_{S}\left(S_{0}\right)$ is fourth order in gauge and Yukawa couplings. Thus the D term contribution to squark and slepton masses (eqn. 32) is naturally the same order as the contribution from messengers given in (eqn. 23), with $\left\langle S_{3}\right\rangle=S_{0}$. The sign of the mass correction is determined by the sign of $\left(Q_{a}^{X} \gamma_{S}\left(S_{0}\right)\right)$. Recall (eqn. 12), $\gamma_{S}=-\frac{1}{2} \frac{\partial \ln \tilde{Z}_{S}}{\partial \ln \mu}$, hence

$$
\gamma_{S}\left(S_{0}\right)=-\frac{1}{2}\left|\bar{S}_{0}\right|^{2} \frac{\partial^{2} \ln \tilde{Z}_{S}}{\partial\left|S_{0}\right|^{2}} \ln \left(\frac{\left|S_{0}\right|}{\left|\bar{S}_{0}\right|}\right)
$$

longer needed to obtain SUSY breaking, as discussed previously by Binetruy and Dudas [2]. In addition, the presence of $\Lambda_{s}$ in the superspace potential (eqn. 31) explicitly breaks $\mathrm{U}(1)_{X}$. In string theories this problem is resolved by the "dilaton." $\Lambda_{s}$ transforms as $\operatorname{det} M$ under $\mathrm{U}(1)_{X}$ due to its implicit dependence on the "dilaton" field $S=1 / g^{2}+i a$ where $a \rightarrow a+\frac{1}{2} \delta_{G S} \theta$ (with $\delta_{G S}=\frac{\operatorname{Tr} \mathbf{Q}^{\mathbf{x}}}{192 \pi^{2}}$ ) under a $\mathrm{U}(1)_{X}$ phase rotation by an angle $\theta$ [2, 3]. Once the "dilaton" is included the anomally for $\mathrm{U}(1)_{X}$ is cancelled a la Green-Schwartz. 
and the coefficient of the logarithm is simply related to the second derivative of the scalar potential $V_{0}$ evaluated at the extremum $\bar{S}_{0}$. Thus at the minimum of the potential the coefficient is positive and if $\left|S_{0}\right|>\left|\bar{S}_{0}\right|$ the logarithm is also positive. We thus see that, under these general conditions, the sign of the $\mathrm{D}$ term correction is determined solely by the $\mathrm{U}(1)_{X}$ charge $Q_{a}^{X}$.

Gauginos obtain no mass from this source. In addition, since the D term contribution to scalar masses (squarks, sleptons and Higgs) is proportional to their $\mathrm{U}(1)_{X}$ charge $Q_{a}^{X}$, in order to suppress flavor changing neutral current interactions the $\mathrm{U}(1)_{X}$ charges of squarks and sleptons must be family independent. One interesting possibility is that $\mathrm{U}(1)_{X}$ is identified with the $\mathrm{U}(1)$

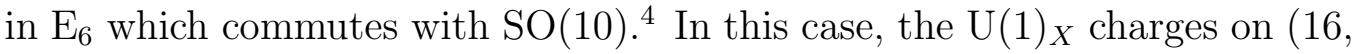
$10,1)$ in the 27 of $\mathrm{E}_{6}$ are given by $(1,-2,4)$. As a consequence, the standard model fermions, in sixteens of $\mathrm{SO}(10)$, have identical positive charge under this $\mathrm{U}(1)_{X}$; thereby obtaining identical positive mass squared corrections. Higgs, on the other hand, in tens of $\mathrm{SO}(10)$, have opposite charge and thus negative mass squared corrections.

The messengers generate SUSY breaking masses for both scalars and gauginos. Their contribution to scalar masses are naturally family independent.

\section{Discussion and Conclusions}

The model as it stands has one problem which is easily fixed. The flavor $\mathrm{SU}(2)_{F}$ gauge symmetry is broken to $\mathrm{U}(1)_{F}$ flavor via the vacuum expectation value of an $\mathrm{SU}(2)_{F}$ vector $\left\langle S_{3}>\right.$. U $(1)_{F}$ remains unbroken at low energies and thus there exists a massless gauge boson, a $\gamma_{F}$. This $\gamma_{F}$ couples to quarks and leptons through loops containing the messengers and standard model gauge interactions. A single $\gamma_{F}$ vertex with ordinary matter is forbidden by $\mathrm{U}(1)_{F}$ charge conjugation. Nevertheless a two $\gamma_{F}$ coupling is allowed. A new massless gauge interaction would probably have been observed. Thus we consider how to break this unwanted $\mathrm{U}(1)_{F}$ symmetry. This is in fact quite easy. We can introduce $\mathrm{SU}(2)_{F}$ doublets into the theory which naturally

\footnotetext{
${ }^{4}$ The possibility of an anomalous $\mathrm{U}(1)_{X}$ derived from strings has recently been considered in the literature [4]. These authors consider the same $\mathrm{U}(1)_{X}$ as discussed here.

${ }^{5}$ Note, $\operatorname{Tr} Q^{X}>0$, as assumed earlier, if 16 s dominate in the sum.
} 
obtain vevs of order the weak scale or larger. Consider introducing $n_{\phi}$ doublet fields $\phi_{\alpha}, \bar{\phi}^{\alpha}$. Assume for the moment they do not enter the superspace potential $W$. Thus at tree level they only enter the D term for the $\mathrm{SU}(2)_{F} .0$ They obtain mass at two loops due to SUSY breaking effects. Recall $S_{3}$ breaks both $\mathrm{SU}(2)_{F}$ and SUSY. As a result the massive gauge sector in $\mathrm{SU}(2)_{F} / \mathrm{U}(1)_{F}$ acts as messengers of SUSY breaking for the $\phi$ s. Using the results of Giudice and Rattazzi 17] (eqns. 61 - 65), we find

$$
m_{\phi}^{2}\left(S_{0}\right)=2 c^{\prime} \frac{\alpha_{F}^{2}}{(4 \pi)^{2}} N\left[1+r\left(\frac{N}{b^{\prime}}-1\right)\right]\left|\frac{F}{M_{m e s s}}\right|^{2}
$$

where $N=b^{\prime}-b ; b^{\prime}$ and $b$ are the coefficients of the $\mathrm{U}(1)_{F}$ and $\mathrm{SU}(2)_{F}$ beta functions; $r=\left(c / c^{\prime}-1\right) /\left(b / b^{\prime}-1\right)$, and $c^{\prime}$ and $c$ are the quadratic Casimirs of the matter gauge representations of the groups $\mathrm{U}(1)_{F}$ and $\mathrm{SU}(2)_{F}$, respectively. In our case $c^{\prime}=1 / 4, c=3 / 4, b^{\prime}=-n_{\phi}$ and $b=2-n_{\phi}$ (see eqn. 17). Thus $N=-2$. The mass squared correction to $\phi$ is negative for $n_{\phi}>1$ and is given by

$$
m_{\phi}^{2}=-\left(n_{\phi}-1\right)\left(\frac{\alpha_{F}}{4 \pi}\right)^{2}\left|\frac{F}{M_{\text {mess }}}\right|^{2}
$$

Now consider $n_{\phi}=2$. At the messenger scale $\phi, \bar{\phi}$ obtain negative mass squared of order the weak scale. The potential for $\phi, \bar{\phi}$ is thus unbounded from below. A contribution to the superspace potential of the form

$$
W=\frac{1}{M}\left(\phi_{\alpha} \bar{\phi}^{\alpha}\right)^{2}
$$

leads to a potential for $\phi, \bar{\phi}$ of the form

$$
V(\phi, \bar{\phi})=-\left|m_{\phi}^{2}\right|\left(|\phi|^{2}+|\bar{\phi}|^{2}\right)+\frac{2}{M^{2}}\left(|\phi|^{2}+|\bar{\phi}|^{2}\right)\left|\phi_{\alpha} \bar{\phi}^{\alpha}\right|^{2}
$$

and to a $\mathrm{U}(1)_{F}$ breaking vev

$$
<\phi>\sim \sqrt{\left|m_{\phi}\right| M} \sim 10^{9-10} \mathrm{GeV}
$$

for $M \sim 10^{16-18} \mathrm{GeV}$. Note, with the additional $n_{\phi}=2$ doublets, the $\mathrm{SU}(2)_{F}$ beta function vanishes at one loop. The only effect this has on our previous results is that now $b=0$ in (eqns. 18, 32) for the induced $\mathrm{D}$ term.

\footnotetext{
${ }^{6}$ We assume they have zero $\mathrm{U}(1)_{X}$ charge.
} 
We have presented a model for SUSY breaking which can provide a phenomenologically interesting spectrum of sparticle masses. Sparticles obtain both $\mathrm{D}$ term (eqns. 18, 32) and gauge mediated messenger contributions (eqn. 23) to their mass. For phenomenological purposes our result suggests using the following form for $\mathrm{D}$ term contributions

$$
\tilde{m}_{a}^{2}=D M_{2}^{2} Q_{a}^{X}
$$

where $Q_{a}^{X}$ is the anomalous $\mathrm{U}(1)_{X}$ charge of particle $a, M_{2}=\frac{\alpha_{2}}{4 \pi} \Lambda$ (wino mass) can be taken to set the scale for this contribution and $D$ is an arbitrary parameter of order one. $Q_{a}^{X}$ is assumed to be family independent. In the minimal model, $Q_{a}^{X}=1$ for quarks and leptons and $Q_{a}^{X}=-2$ for Higgs doublets. The model has two fundamental scales. The SUSY breaking scale $\sqrt{F_{S_{3}}}=\Lambda_{s}$ is the dynamical scale of a strong $\mathrm{SU}(2)$ gauge symmetry. The messenger scale $<S_{3}>=S_{0}$ is set by the Fayet-Iliopoulos $\mathrm{D}$ term. In strings the latter scale is fixed by the compactification scale. The effective SUSY breaking scale in the observable sector is given by $\Lambda=\frac{2 F_{S_{3}}}{\left\langle S_{3}\right\rangle} \approx 10^{5}$ $\mathrm{GeV}$. We assume that $S_{0}$ is bound from above by $\sim 10^{15} \mathrm{GeV}$ in order to suppress gravity mediated SUSY breaking masses which are not gauranteed to be family independent. The natural scale for $S_{0}$ (eqn. 28) is of order $\sim M_{s t} / 10$. Thus the scenario presented in this paper would best fit into the strong coupling limit of the heterotic string 18 in which $M_{s t}$ is identified with the GUT scale $\sim 10^{16} \mathrm{GeV}$.

A recent paper on the cosmological problems associated with gravitinos has found extremely low reheat temperatures are necessary for weak scale gauge mediated models with very light gravitinos [19]. In our case, the gravitino mass is of order $12 \mathrm{GeV}$ and the reheat temperature is much higher.

Finally it has been argued that, with a particular mechanism for stabilizing the dilaton in string theories, the dilaton SUSY breaking contribution always dominates over the D term SUSY breaking contribution of an anomalous $\mathrm{U}(1)_{X}[3]$. If this is true, then the SUSY breaking contributions considered in this paper are subdominant. However, other mechanisms for stabilizing the dilaton may not have this effect, see for example [20] where the dilaton is stabilized by contributions to the superspace potential. In this case dilaton SUSY breaking vanishes. Clearly, this potential dilaton problem requires further study.

Acknowledgements Finally, this work is partially supported by DOE grant DOE/ER/01545-740. 


\section{References}

[1] G. Dvali and A. Pomarol, Phys. Rev. Lett. 77, 3728 (1996); G. Dvali and A. Pomarol, "Anomalous U(1), Gauge-Mediated Supersymmetry Breaking and Higgs as Pseudo-Goldstone Bosons," hep-ph/9708364.

[2] P. Binetruy and E. Dudas, Phys. Lett.B389, 503 (1996).

[3] N. Arkani-Hamed, M. Dine and S.P. Martin, "Dynamical supersymmetry breaking in models with a Green-Schwarz mechanism," hepph/9803432.

[4] A.E. Faraggi and J.C. Pati, "A Family-Universal Anomalous U(1) in String Models as the Origin of Supersymmetry Breaking and Squark Degeneracy," hep-ph/9712516.

[5] L.E. Ibanez and G.G. Ross, Phys. Lett.B332, 100 (1994).

[6] P. Binetruy and P. Ramond, Phys. Lett.B350, 49 (1995); P. Binetruy, S. Lavignac and P. Ramond, Nucl. Phys.B477, 353 (1996); P. Binetruy, N. Irges, S. Lavignac and P. Ramond, Phys. Lett.B403, 38 (1997); J.K. Elwood, N. Irges, and P. Ramond, Phys. Lett.B413, 322 (1997); N. Irges, S. Lavignac and P. Ramond, "Predictions from an Anomalous U(1) Model of Yukawa Hierarchies," hep-ph/9802334.

[7] V. Jain and R. Shrock, Phys. Lett.B352, 83 (1995).

[8] E. Dudas, S. Pokorski and C.A. Savoy, Phys. Lett.B356, 45 (1995); E. Dudas, C. Grojean, S. Pokorski and C.A. Savoy, Nucl. Phys.B481, 85 (1996).

[9] E.J. Chun and A. Lukas, Phys. Lett.B387, 99 (1996); K. Choi, E.J. Choi and H. Kim, hep-ph/9611293.

[10] R.N. Mohapatra and A. Riotto, Phys. Rev.D55, 1137 (1997) and Phys. Rev.D55, 4262 (1997).

[11] A.E. Nelson and D. Wright, "Horizontal anomalous U(1) symmetry for the minimal supersymmetric standard model," hep-ph/9702359.

[12] N. Seiberg, Phys. Rev.D49, 6857 (1994) . 
[13] K. Intriligator and S. Thomas, Nucl. Phys.B473, 121 (1996). ; K.-J. Izawa and T. Yanagida, Prog. Theor. Phys.95, 829 (1996).

[14] N. Arkani-Hamed, J. March-Russel and H. Murayama, Nucl. Phys. B509, 3 (1998). ; H. Murayama, Phys. Rev. Lett. 79, 18 (1997) ; S. Dimopoulos, G. Dvali, G. Giudice and R. Rattazzi, Nucl. Phys. B510, 12 (1998).

[15] P. Fayet and J. Iliopoulos, Phys. Lett.B51, 461 (1974) ; W. Fischler, H.P. Nilles, J. Polchinski, S. Raby and L. Susskind, Phys. Rev. Lett.47, 757-759 (1981).

[16] M. Dine, N. Seiberg and E. Witten, Nucl. Phys. B289, 589 (1987) ; J. Atick, L. Dixon and A. Sen, Nucl. Phys. B292, 109 (1987); M. Dine, I. Ichinose and N. Seiberg, Nucl. Phys. B293, 253 (1988) .

[17] G.F. Giudice and R. Rattazzi, Nucl. Phys.B511, 25-44 (1998).

[18] Horava and E. Witten, Nucl. Phys. B460, 506 (1996).

[19] T. Moroi, H. Murayama and M. Yamaguchi, Phys. Lett. B303, 289 (1993); A. de Gouvea, T. Moroi and H. Murayama, Phys. Rev. D56, 1281 (1997).

[20] B. de Carlos, J.A. Casas and C. Munoz, Nucl. Phys. B399, 623 (1993). 